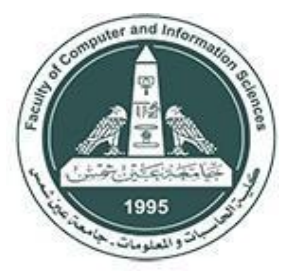

International Journal of Intelligent Computing and Information Sciences

https://ijicis.journals.ekb.eg/

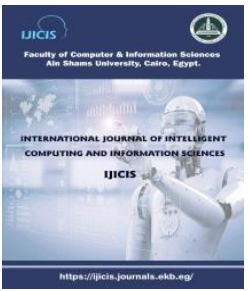

\title{
Comparative Study and Analysis of Recent Computer Aided Diagnosis Systems for Masses Detection in Mammograms
}

\author{
Ghada Hamed* \\ Scientific Computing department, \\ Computer and Information \\ Science, Ain Shams University, \\ Cairo, Egypt \\ ghadahamed@cis.asu.edu.eg \\ Safaa Amin El-Sayed \\ Scientific Computing department, \\ Computer and Information \\ Science, Ain Shams University, \\ Cairo, Egypt \\ safaa.ameen@cis.asu.edu.eg
}

Mohammed Marey

Scientific Computing department Computer and Information Science,

Ain Shams University, Cairo, Egypt

mohammedmarey11@gmail.com

Mohamed Fahmy Tolba

Scientific Computing department, Computer and Information Science,

Ain Shams University, Cairo, Egypt

fahmytolba@gmail.com

Received 2021-1-3; Revised 2021-2-28; Accepted 2021-3-3

Available online 2021-3-4

\begin{abstract}
Nowadays, breast cancer is considered one of the most threatening and common cancers for women due to the high rate of deaths that occurred yearly that reaches about $25 \%$ in all cancers. One of the most keys to decrease the mortal rate caused by breast cancer is its early detection. So, the research on developing computer-aided diagnosis systems (CADs) has been widely increased to improve the accuracy of breast cancer localization and classification. Generally, a proposed CAD is developed through four stages: data preparation and preprocessing, cancer detection, followed by its pathology classification. In this paper, the most recent proposed approaches to detect lesions in the breast mammograms and classify them are discussed with a comparative analysis to list the advantages and the disadvantages of most approaches. The main objective of this paper is to group the CADs with a performance evaluation and detailed analysis in order to furtherly develop others by avoiding the main weak points in the existing systems and to achieve high detection accuracy and classification performance at the same time.
\end{abstract}

* Corresponding author: Ghada Hamed

Scientific Computing department, Computer and Information Science, Ain Shams University, Cairo, Egypt

E-mail address: ghadahamed@ cis.asu.edu.eg 
Keywords: Breast cancer detection, Masses classification, YOLO for masses localization, Full field digital mammograms.

\section{Introduction}

Cancers spread all overworld and one of the most common cancers is the breast cancer especially among females [1]. Breast cancer begins to appear in specific various parts of the breast as lobules, connective tissues or ducts and then it starts to affect other body parts outside the breast results in almost cases to death.

Breast cancer screening is the process of checking women's breasts for cancer if either they complain from any existing symptoms of cancer or even just periodic checkup. The screening process and the periodic checking is one from the main methods results in revealing cancer in breast before spreading in other parts surrounding it or the whole body. Also, CAD system is considered a second reader with radiologists' decision since the process of reading mammograms becomes hard due to increasing the mammograms number taken daily [1-3].

Mammography is the most common process and best way done for the breast cancer diagnosis and screening using low dose of X-rays to check the whole women's breast to early detect the breast cancer. The benefit of mammograms over the Breast Magnetic Resonance Imaging (MRI) is that MRI usually used to discover high levels for breast cancer which may results in abnormal results even when there is no cancer. Unlike mammograms which are used in all cases and do not bias to specific severity of breast cancer.

Since mammograms may view some existing tumors as false negatives results in late discover for the cancer and consequently late diagnosis and late treatment, Computer Aided Diagnosis (CAD) is developed to act as a second eye besides the radiologist's decision.

Large number of CAD systems are proposed using different machine learning models and now designed using various deep learning models to detect accurately any existing cancers in the screened mammogram for early diagnosis and treatment [4-6].

So, this paper mainly introduces a complete survey for the most recent state of art for the developed CADs used in the breast cancer localization and classification as well [7,8]. This paper aims as well to be a starting point for proposing better cancer detection accuracy based on the presented work that is introduced in form of many different views [9-13].

The paper is structured as follows: the public available datasets is represented in section 2 with a detailed comparison between them. Then, in section 3, the evaluation metrics used for measuring the detection and the classification performance are listed. In section 4, the most recent work introduced in the breast cancer detection and classification is discussed with a comparative analysis between them in addition to their advantages and disadvantages. Finally, in section 5, the work conclusion and other suggested future work have been introduced.

\section{Mammograms Datasets}

In this section, different types for mammograms are discussed besides the datasets contain mammograms and exist publicly on web with a detailed comparison between the features of each. 


\subsection{Mammograms Types}

The mammograms are existing in two formats either in Fully field digital mammograms (FFDM) or scanned mammograms.

1. FFDM are digital breast mammograms obtained by screening breast through some few dose of the X-rays to be converted into electric signals. These generated mammograms can be checked on a computer screen or printed on a film to be scanned to ordinary mammograms.

2. Scanned mammograms and those generated from the breast scanning machine directly. Then, passed through scanners to be converted to semi-digital mammograms for further checking by CADs on computers screens.

\subsection{Mammograms Views}

During the screening process, left and right breasts can be examined from two views which are either cranio-caudal (CC) and mediolateral-oblique (MLO). A sample for each case is given in Figure 1. The $\mathrm{CC}$ view is screening the breast from above as shown in (a) and (b) in Figure 1 while the MLO is taken from an angled view as shown in (c) and (d). The MLO is the preferable view for radiologists since in the MLO view, more of the breast details in the upper outer quadrant is shown.

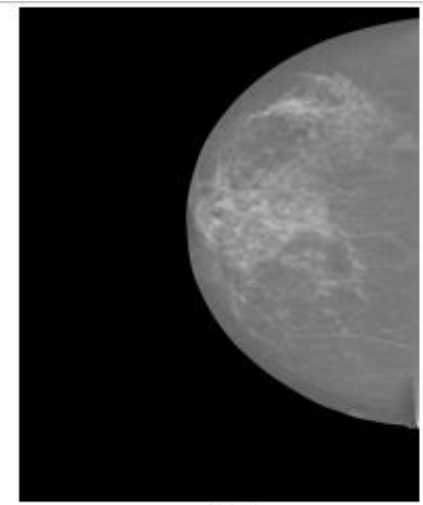

(a)

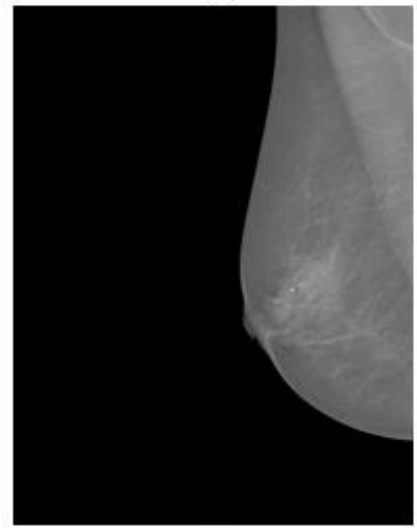

(c)

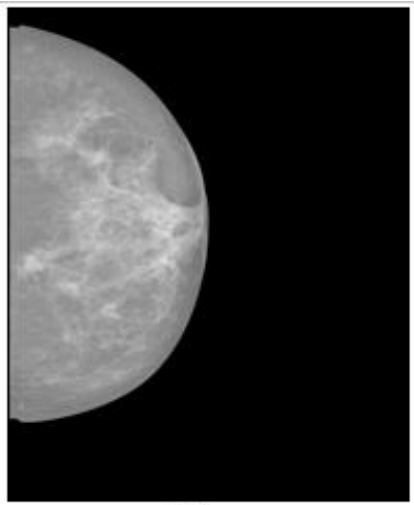

(b)

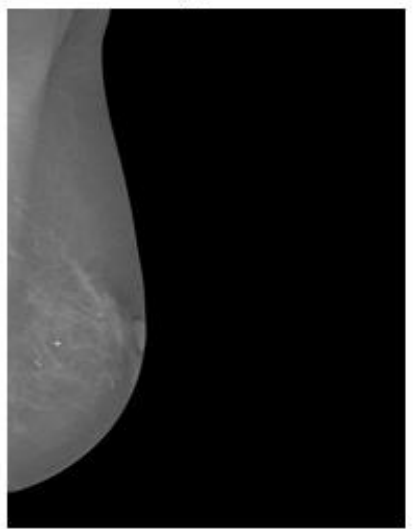

(d)

Figure 1: Samples from the INbreast dataset for CC and MLO views. (a) Right CC mammogram,

(b) Left CC mammogram, (c) Right MLO mammogram, (d) Left MLO mammogram 


\subsection{Public Datasets}

There are many available datasets of breast mammograms on internet. Some of them are FFDM or scanned mammograms, contain masses or calcifications or both. In this section, most of the public and commonly used datasets are listed with the properties of each and the main differences between them. They are:

- INbreast. The INbreast dataset contains FFDM mammograms acquired at a Breast Centre, located in a University Hospital [14]. It contains a total of 410 mammograms of 115 different cases. Some of the cases are obtained from women with their two breasts, i.e., four mammograms for each case of women. These cases are 90 cases and the other remaining 25 cases are obtained from mastectomy patients, i.e., two mammograms per one case.

The INbreast contains different types of lesion that may exist in breast such as masses, calcifications, distortions of asymmetries. Each case exists with the bounding box annotations of the existing lesions besides the type of the lesion and its pathology type. All annotations and contours are given in XML files that are prepared and confirmed by specialists in this field. This is the only dataset that is considered public to be obtained and contains fully field digital mammograms as well.

- DDSM. The Digital Database for Screening Mammography (DDSM) is a set of scanned film mammograms collected by Massachusetts General Hospital (D. Kopans, R. Moore), the University of South Florida (K. Bowyer), and Sandia National Laboratories (P. Kegelmeyer) [15]. It contains 2620 different cases. Each case is obtained from the two breasts of a specific patient, i.e., two mammograms for each breast.

The dataset includes the ground truth for each mammogram in terms of the lesions bounding box coordinates, some details for the study itself as its time and patient age besides the suspicious regions types, the breast density rate, the mass pathology and the subtlety rating for the existing abnormalities.

- CBIS-DDSM. It is a newer version from the DDSM [16][17]. The Curated Breast Imaging Subset of DDSM (CBIS-DDSM) contains compressed mammograms in form of DICOM format. All annotations are revised and updated by some specialists to get more accurate region of interest (ROI) segmentation and bounding boxes annotations besides the pathology type of any abnormalities in the breast.

- MIAS. The Mammographic Image Analysis Society (MIAS) organization worked to get MIAS dataset of different digital mammograms [18]. The mammograms are film images that are obtained from the UK national breast screening program to get digitized images. It contains 322 mammograms with the location of all existing abnormalities.

A detailed comparison has been introduced between the mentioned public datasets in Table 1. For each dataset, the types of its mammograms have been mentioned if fully field digital mammograms (FFDM) or scanned film mammograms (SF). Also, the number of cases per dataset and the number of mammograms result from these cases have been listed in the table. Also, the sizes of the mammograms 
exist, and their formats are included in the comparison as well if either Joint Photographic Experts Group (JPEG), Digital Imaging and Communications in Medicine (DICOM) or Portable Grayscale Map (PGM).

An example for mammogram samples of each dataset is shown in Figure 2 and the advantages and the disadvantages of each dataset have been listed in

Table 2.

Table 1: Comparison between the public datasets contain mammograms of breast cancer (MT: Mammograms Type, \#_C:

Number of Cases, \#_M: Number of mammograms, MS: Mammograms Sizes, MF: Mammograms Format)

\begin{tabular}{|c||c|c|c|c|c|}
\hline Dataset & MT & \#_C & \#_M & MS & MF \\
\hline \hline INbreast & FFDM & 115 & 410 & $\begin{array}{c}3328 * 4084 \text { or } \\
2560 * 3328\end{array}$ & DICOM \\
\hline DDSM & SF & 2620 & 10,480 & $3000 * 4800$ & JPEG \\
\hline CBIS-DDSM & SF & 6775 & 10,239 & Not fixed sizes & DICOM \\
\hline MIAS & SF & - & 322 & $1024 * 1024$ & PGM \\
\hline
\end{tabular}

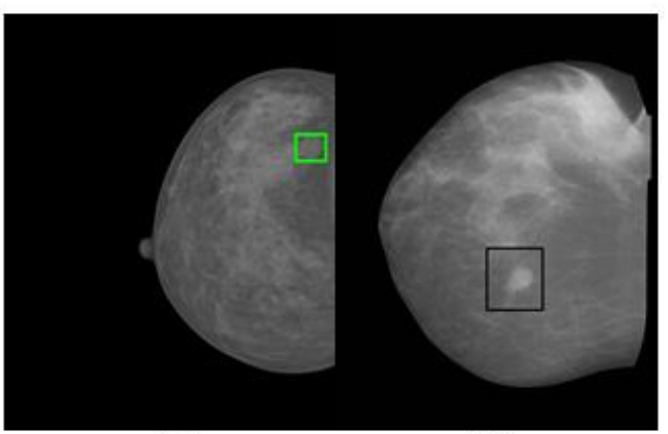

(a)

(b)

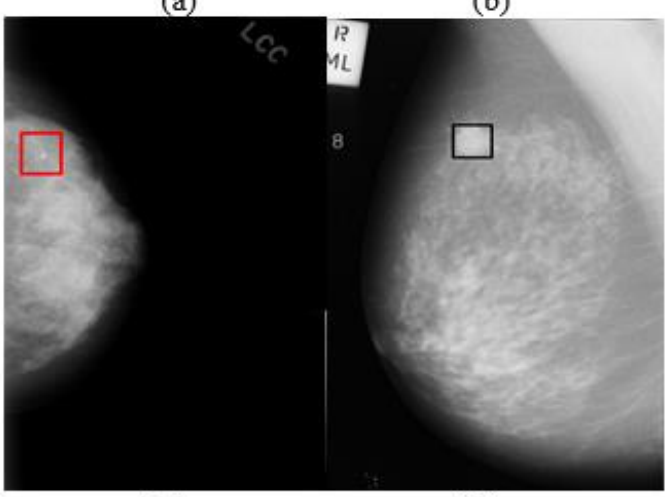

(c)

(d)

Figure 2: Datasets Samples with their ground truth; (a) A mammogram sample from the INbreast,

(b) A mammogram sample from the DDSM, (c) A mammogram sample from the CBIS-DDSM and (d) A mammogram sample from the MIAS dataset 
Table 2: Advantages and disadvantages of each dataset

\begin{tabular}{|c|c|c|}
\hline Dataset & Advantages & Disadvantages \\
\hline INbreast & $\begin{array}{l}\text { - } \text { Mammograms are FFDM. } \\
\text { - Very good quality for the } \\
\text { included cases. } \\
\text { - } \quad \text { Accurate annotations. } \\
\text { - } \quad \text { Contain normal cases. }\end{array}$ & $\begin{array}{l}\text { - Small dataset. } \\
\text { - } \text { Unbalanced classes. }\end{array}$ \\
\hline DDSM & $\begin{array}{l}\text { - Large number of cases and } \\
\text { mammograms. }\end{array}$ & $\begin{array}{l}\text { - } \text { Bad quality. } \\
\text { - } \quad \text { A lot of noise per mammograms. } \\
\text { - } \quad \text { Inaccurate annotations. }\end{array}$ \\
\hline CBIS-DDSM & $\begin{array}{l}\text { - Large number of cases and } \\
\text { mammograms. } \\
\text { - Well organized categories. } \\
\text { - Accurate annotations. }\end{array}$ & $\begin{array}{l}\text { Mammograms are scanned film } \\
\text { which results in lower } \\
\text { performance than FFDM. }\end{array}$ \\
\hline MIAS & $\begin{array}{l}\text { - Different classes exist. } \\
\text { - It contains lesions with different } \\
\text { sizes and properties which } \\
\text { results in different varieties. }\end{array}$ & $\begin{array}{l}\text { - Small dataset. } \\
\text { - Unbalanced classes. } \\
\text { - } \text { Bad quality. }\end{array}$ \\
\hline
\end{tabular}

As shown since the INbreast is the only dataset contains fully field digital mammograms, the given sample is the best from the quality wise compared with other samples of the other databases and it is the one with the lowest quantity of noise on mammogram that may result from the process of the breast scanning. The disadvantage of the DDSM dataset, is the inaccurate annotations given for some mammograms such that in many cases the bounding box represents the ground truth of the existing lesion is somehow larger than or smaller than the actual region of interest occupied the mass. This DDSM problem has been solved in the CBIS-DDSM dataset that is updated with more accurate annotations for the ground truth. All the mentioned datasets contain both views of mammograms CC or MLO for both left and right breasts.

\section{Evaluation Metrics}

The commonly used performance evaluation metrics to evaluate the mass detection and the classification performance are listed in this section.

\subsection{Detection Performance Metrics}

To evaluate how the localization of the mass is accurate and matches the actual mass's ground truth, the metrics used are:

- Intersect Over Union (IOU): It is used to determine if the lesion location is localized correctly or not. It is the intersection area divided over the union area of two bounding boxes. Therefore, IOU must have a value larger than or equal to zero and smaller than or equal to one. 
Usually from the literature review it is conducted that the most published work for determining the mass position in mammogram is detected in case of IOU higher than $50 \%$ compared with the coordinates of its ground truth.

\subsection{Classification Performance Metrics}

The classification accuracy is measured mainly using the confusion matrix which is a matrix that evaluates the accuracy of a specific classification model based on the testing set. The number of classes usually is two named benign and malignant (binary classification). So, the confusion matrix for a binary classifier measures the following four items:

- True Positives (TP): These are cases that are predicted as malignant, and they do have malignant mass.

- True Negatives (TN): These are cases that do have benign mass and they are predicted as negative or benign.

- False Positives (FP): These are cases that are predicted as malignant, but they do not actually have malignant mass.

- False Negatives (FN): These are cases that are predicted as benign, but they actually do have malignant mass.

For example, given 100 mammograms and 100 masses exist in these mammograms with 2 classes: benign and malignant. There are 90 masses that are classified as malignant (marked as 1 to represent the positive class), and 10 masses are classified as benign (marked as 0 to represent the negative class).

Now let us assume that these images are the input of a model, and the model classified 85 malignant masses as malignant, 1 benign mass as benign, 9 benign masses as malignant and 5 malignant masses as benign. In this case, $\mathrm{TP}$ is $85, \mathrm{TN}=1, \mathrm{FP}=9$ and $\mathrm{FN}=5$.

These four items are used to compute the main metrics used to measure the mass classification performance. They are precision, true positive, false positive, true negative, the overall model's accuracy, and the mean average precision. They are computed as follows:

1. Precision: It is the ratio of the true positives and the total predictions. It measures when model PREDICTS yes for malignant masses, how often is it correct? Its formula is given as follows:

$$
\text { Precision }=\frac{\mathrm{TP}}{\mathrm{TP}+\mathrm{FP}}
$$

So, for the precision computation of this classifier, the 100 masses classified by the model shall be checked. If 9 of them are wrongly classified (not malignant or related to the positive class $)$, then the precision will be $=85 /(85+9)=0.90$.

2. True Positive Rate (TPR/Recall/Sensitivity): It is the ratio of the true predicted objects and the number of the ground truths represent the positive predictions. It measures when it's 
ACTUALLY malignant, how often does it PREDICTS malignant? Its formula is given as follows:

$$
\text { Recall }=\frac{\mathrm{TP}}{\text { Actual Malignant }}=\frac{\mathrm{TP}}{\mathrm{TP}+\mathrm{FN}}
$$

For our example the recall $=85 /(85+5)=0.94$. So, the recall is used to evaluate how good all the masses in the data are detected correctly.

3. False Positive Rate (FPR): It measures when it is ACTUALLY benign, how often does it PREDICTS malignant? Its formula is given as follows:

$$
\mathrm{FPR}=\frac{\mathrm{FP}}{\text { Actual Benign }}=\frac{\mathrm{FP}}{\mathrm{TN}+\mathrm{FP}}
$$

On our example the FPR $=9 /(1+9)=0.90$.

4. True Negative Rate (TNR/Specificity): It measures when it is ACTUALLY benign, how often does it PREDICTS benign? Its formula is given as follows:

$$
\text { Specificity }=\frac{\mathrm{TN}}{\text { Actual Benign }}=\frac{\mathrm{TN}}{\mathrm{TN}+\mathrm{FP}}
$$

Then, TNP for the proposed example $=1 /(1+9)=0.10$.

5. Overall Accuracy: It measures the overall accuracy of the used classifier as follows:

$$
\text { Overall Accuracy }=\frac{\mathrm{TP}+\mathrm{TN}}{\text { Total Actual }}=\frac{\mathrm{TP}+\mathrm{TN}}{\mathrm{TP}+\mathrm{FN}+\mathrm{TN}+\mathrm{FP}}
$$

So, the overall accuracy of the given example $=(85+1) /(85+1+9+5)=0.86$.

6. Average Precision (AP) and mean Average Precision (mAP): The AP is the area under the precision-recall curve by combining recall and precision as shown in Figure 3. While the mAP is the AP mean computed for the given classes. The mAP is calculated as follows:

$$
m A P=\frac{\sum_{q=1}^{Q} A v e P(q)}{Q}
$$




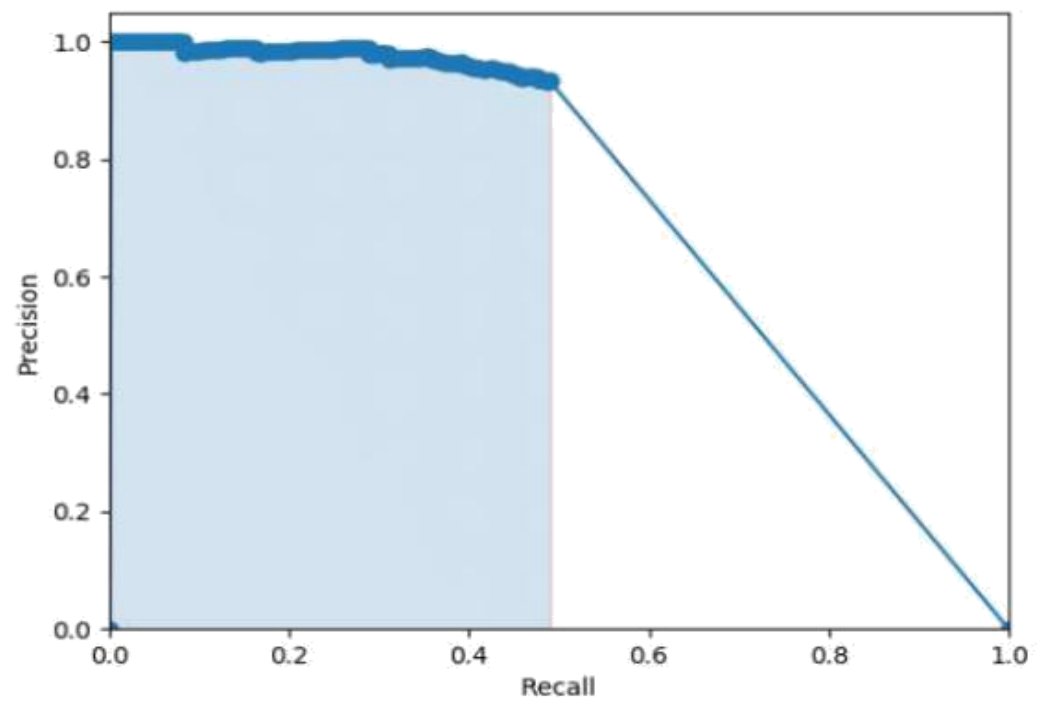

Figure 3: Precision Recall Curve

\section{Detection \& Classification Methods}

Since the early detection for the breast cancer is the main key to decrease the mortal rate among women, the research in the new era is going towards improving the detection performance of any lesions can exist in mammograms by developing new CAD based on AI.

In general, the main flow to reveal cancer as shown in Figure 4, begins with the mammograms preprocessing to prepare the images to be in the format required by the model responsible for the features extraction and the masses detection process, also the masses annotations are extracted from the input regions of interest that represent the actual ground truth.

Then, the masses detection phase starts by learning from the given features of the training set to be ready to predict the region of any existing masses in the testing set. Finally, some CADs are developed as end-to-end systems to classify the extracted lesions from the mammograms if benign or malignant or normal mammogram. Not all CAD systems contain the three steps shown in Figure 4. 


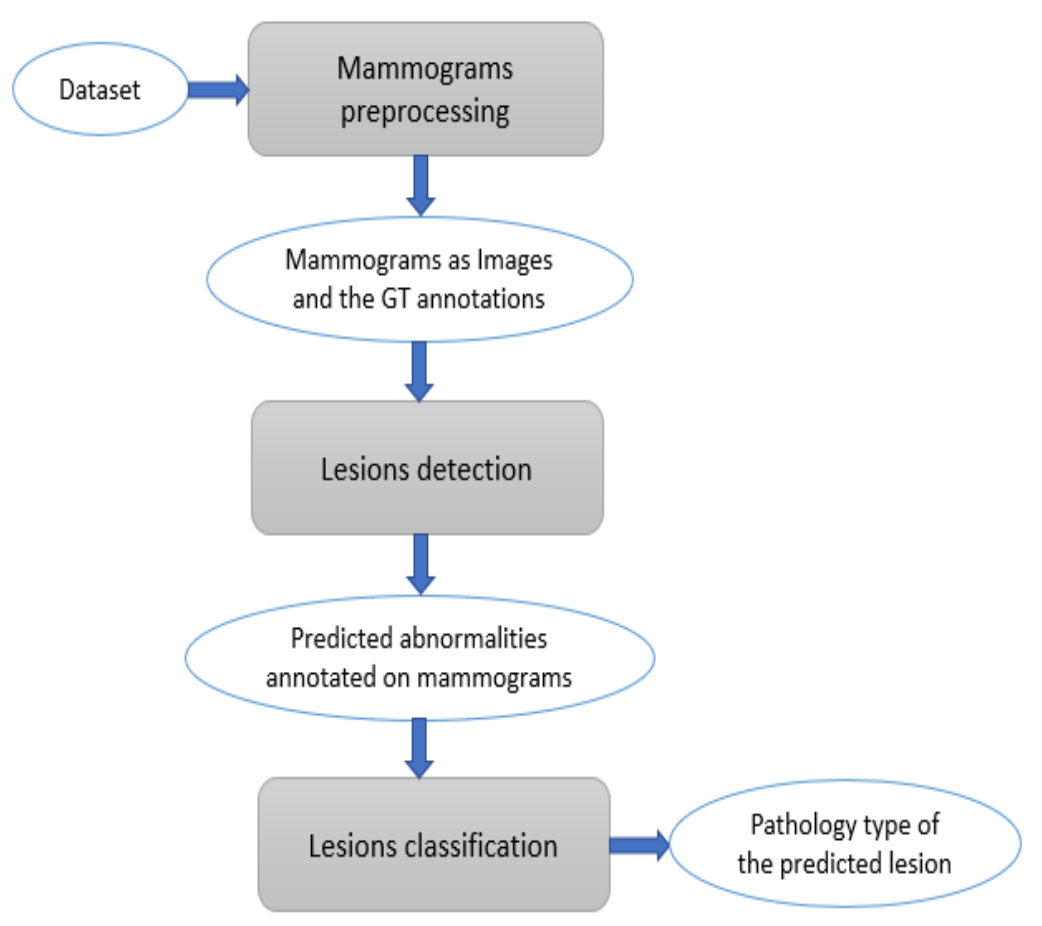

Figure 4: Block diagram of CADs for detecting breast cancer

In this section, the most recent published work to localize lesions in breast and then classify them accurately, are compared. Some models are proposed to only detect abnormalities in mammograms or to only classify the detected lesions if benign or malignant or both as will be shown later in the next subsections. The eight recent methods used to either detect lesions in mammograms or classify them or both are analysed in Error! Reference source not found. and Error! Reference source not found. to investigate their advantages and disadvantages in order to avoid in any further proposed work.

\subsection{Mammograms Preprocessing}

In almost cases, mammograms pre-processing is essential step to remove various forms or noises. Noises can be represented on mammograms in form of dust obtained through the scanning process or any extra move in the breast scanning device. Also, in case of the scanned mammograms, different forms of noise can be represented in form of any labels on the mammograms or any information or details about the patient or the case itself.

So, in almost cases especially the scanned mammograms more than the fully field digital mammograms, the pre-processing step becomes very important to be conducted.

Data augmentation is considered a step from the pre-processing steps to achieve higher performance in the detection accuracy especially if the dataset is small or does not contain many varieties. Data augmentation is increasing the samples of the training set by applying geometric transformations such as applying rotation, flipping on the original samples using simple image processing techniques.

\subsection{Lesions Detection}

Detection of the abnormalities in breast mammograms is the process of extracting the regions of interest represent any lesions. This process can be held either by image segmentation or by utilizing the transfer 
learning on different deep learning approaches to learn the features of masses can be exist in mammograms. Different methods are implemented using different models and different datasets in order to localize lesions in breast mammograms as mentioned in Table 3 and Table 4 in the fourth column of the detection method with the detection accuracy of each.

Table 3: Comparative study among the most recent proposed methods for the breast cancer detection and classification (DA: Refers to the Detection Overall Accuracy, CA: Refers to the Classification Overall Accuracy, and the IOU used by all of them $=0.5$ ) - PART 1

\begin{tabular}{|c|c|c|c|c|c|c|}
\hline $\mathbf{M}$ & Dataset & Pre-processing & Detection & Classification & DA (\%) & $\mathrm{CA}(\%)$ \\
\hline [19] & $\begin{array}{l}\text { Private dataset } \\
\text { of } 72 \text { right and } \\
\text { left breast of } 18 \\
\text { patients and } \\
152 \text { views of } \\
\text { normal } 38 \\
\text { patients. }\end{array}$ & $\begin{array}{l}\text { All mammograms are } \\
\text { enhanced using median } \\
\text { filtering. }\end{array}$ & $\begin{array}{l}\text { Morphological } \\
\text { operations are done } \\
\text { to segment the } \\
\text { tumour region from } \\
\text { the mammogram. }\end{array}$ & Random forest & - & 95.3 \\
\hline [20] & $\begin{array}{l}\text { Private dataset } \\
\text { of } 9109 \\
\text { microscopic } \\
\text { photographs of } \\
\text { breast cancer } \\
\text { tissue. }\end{array}$ & $\begin{array}{l}\text { All mammograms are } \\
\text { resized into } 224 * 224 \text {. }\end{array}$ & $\begin{array}{l}\text { An enhanced } \\
\text { adaptive learning } \\
\text { based Gaussian } \\
\text { Aggregate Model } \\
\text { (GMM) is used to } \\
\text { allocate and segment } \\
\text { ROI. }\end{array}$ & $\begin{array}{l}\text { A Radial Basis } \\
\text { Function (RBF) } \\
\text { kernel based } \\
\text { SVM classifier is } \\
\text { used to classify } \\
\text { the segmented } \\
\text { masses. }\end{array}$ & - & 96.0 \\
\hline [21] & $\begin{array}{l}\text { Private dataset } \\
\text { of } 8000 \\
\text { mammograms }\end{array}$ & $\begin{array}{l}\text { Data is augmented by } \\
\text { using different } \\
\text { transformations as } \\
\text { translating, rotation, } \\
\text { flipping and scaling. }\end{array}$ & $\begin{array}{l}\text { Features extracted } \\
\text { from mammograms } \\
\text { using three CNN } \\
\text { architectures of } \\
\text { GoogLeNet, } \\
\text { VGGNet and ResNet } \\
\text { are combined using } \\
\text { transfer learning } \\
\text { concept. }\end{array}$ & $\begin{array}{l}\text { Average pooling } \\
\text { is used in a fully } \\
\text { connected } \\
\text { network to } \\
\text { classify detected } \\
\text { objects into } \\
\text { benign and } \\
\text { malignant. }\end{array}$ & - & 97.52 \\
\hline [22] & DDSM & $\begin{array}{l}\text { Select } 600 \text { cases. } \\
\text { Each mammogram is } \\
\text { augmented through } \\
\text { rotating it by } 3 \text { different } \\
\text { angles. }\end{array}$ & $\begin{array}{l}\text { You Only Look Once } \\
\text { (YOLO-V1) }\end{array}$ & YOLO-V1 & 99.7 & 97.0 \\
\hline
\end{tabular}

\subsection{Lesions Classification}

Based on the selected regions of interest represent abnormalities in mammograms, the suspicious bounding box of the detected lesion is classified as benign or malignant using various classification methods. In Table 3 and Table 4, the most recent classification methods are listed. Usually, the classification process is occurred to the only true positive objects localized using a specific detector.

Table 4: Comparative study among the most recent proposed methods for the breast cancer detection and classification (DA: Refers to the Detection Overall Accuracy, CA: Refers to the Classification Overall Accuracy, and the IOU used by all of them $=0.5$ ) - PART 2

\begin{tabular}{|c||c|c|c|c|c|c|}
\hline M & Dataset & Pre-processing & Detection & Classification & DA (\%) & CA (\%) \\
\hline \hline
\end{tabular}




\begin{tabular}{|c|c|c|c|c|c|c|}
\hline [23] & DDSM & $\begin{array}{l}\text { Multi-threshold } \\
\text { peripheral equalization } \\
\text { technique is applied on } \\
\text { mammograms to } \\
\text { remove the effect of } \\
\text { breast compression. } \\
\text { Mammograms are } \\
\text { resized to } 448 * 448 \text {. }\end{array}$ & $\begin{array}{l}\text { You Only Look Once } \\
\text { (YOLO) }\end{array}$ & $\begin{array}{l}\text { Fully connected } \\
\text { neural network } \\
(\text { FC-NN) }\end{array}$ & 96.33 & 85.52 \\
\hline [24] & $\begin{array}{l}\text { DDSM \& } \\
\text { INbreast }\end{array}$ & $\begin{array}{l}\text { All mammograms are } \\
\text { rotated eight times } \\
\text { around the origin. } \\
\text { Left-right and up-down } \\
\text { Flipping are applied for } \\
\text { each mammogram. }\end{array}$ & $\begin{array}{l}\text { You Only Look Once } \\
\text { (YOLO-V1) }\end{array}$ & $\begin{array}{l}\text { feedforward } \\
\text { CNN, ResNet- } \\
50 \text {, and } \\
\text { InceptionResNet } \\
\text {-V2 }\end{array}$ & $\begin{array}{l}\text { DDSM: } \\
99.17 \\
\text { INbreast: } \\
97.27\end{array}$ & $\begin{array}{c}\text { DDSM } \\
\text { CNN: } 94.50 \\
\text { ResNet-50: } \\
95.83 \\
\text { InceptionRes } \\
\text { Net-V2: } \\
97.50 \\
\text { INbreast } \\
\text { CNN: } 88.74 \\
\text { ResNet-50: } \\
\text { 92.55 } \\
\text { InceptionRes } \\
\text { Net-V2: } \\
\text { 95.32 }\end{array}$ \\
\hline [25] & INbreast & $\begin{array}{l}\text { Data is augmented by } \\
\text { rotating each } \\
\text { mammogram } 3 \text { angles. }\end{array}$ & $\begin{array}{l}\text { YOLO You Only } \\
\text { Look Once } \\
\text { (YOLO-V3) }\end{array}$ & $\begin{array}{l}\text { YOLO-V3, } \\
\text { ResNet, } \\
\text { InceptionV3 }\end{array}$ & 89.4 & $\begin{array}{l}\text { ResNet: } 91.0 \\
\text { InceptionV3: } \\
\quad 95.5\end{array}$ \\
\hline [26] & CBIS-DDSM & $\begin{array}{l}\text { Mammograms are } \\
\text { augmented by rotating } \\
\text { each one by } 90,180 \text { and } \\
270 \text { and the same is } \\
\text { done for the flipped } \\
\text { version of each ROI. } \\
\text { High frequency noise is } \\
\text { removed using gaussian } \\
\text { filter. }\end{array}$ & - & $\begin{array}{l}\text { Extracting } \\
\text { features from the } \\
\text { ROIs represent } \\
\text { lesions using the } \\
\text { deep } \\
\text { convolutional } \\
\text { neural network } \\
\text { (DCNN). }\end{array}$ & - & 76.0 \\
\hline
\end{tabular}

\subsection{Comparative Analysis}

Based on the conducted survey done in revealing breast cancer in mammograms, the 8 methods shown in Table 3 and Table 4 with their advantages and disadvantages in Table 5 are selected based on their publication date and the most effective. Some of these methods used private datasets as [19-21] which are obtained from some labs or hospitals. Others from the mentioned used the DDSM which are [22, 23] since it is considered the most public scanned mammograms dataset before publishing the CBISDDSM.

The authors in [24] used a combination from the DDSM and some FFDM from the INbreast and in [25] the INbreast only is used. Since the CBIS-DDSM is considered still new, so it is not used a lot. In this comparative analysis, the proposed method in [26] used the CBIS-DDSM in its training and evaluation process. 
Table 5: Advantages and disadvantages of the recent state of art in the breast cancer detection and classification

\begin{tabular}{|c|c|c|}
\hline Method & Advantage & Disadvantage \\
\hline [19] & $\begin{array}{l}\text { Utilizing the GLCM, entropy and mean } \\
\text { features to examine the texture of the } \\
\text { image, makes a noticeable change in } \\
\text { enhancing the classification performance. }\end{array}$ & Small and unbalanced dataset \\
\hline$[20]$ & $\begin{array}{l}\text { Large dataset. } \\
10 \text {-fold cross validation which results in } \\
\text { reliable and fair results. }\end{array}$ & $\begin{array}{l}\text { It is considered slow compared with the other } \\
\text { models. } \\
\text { Not end to end system, the detection and the } \\
\text { classification are two separate phases. }\end{array}$ \\
\hline$[21]$ & $\begin{array}{l}\text { Utilizing the benefit of transfer learning } \\
\text { to combine multiple features extractors to } \\
\text { enhance the detection and the } \\
\text { classification accuracy. }\end{array}$ & $\begin{array}{l}\text { Slow detection time compared with others. } \\
\text { Not end to end system. }\end{array}$ \\
\hline$[22]$ & $\begin{array}{l}\text { End to end system. } \\
\text { Utilizing YOLO (You Only Look Once) } \\
\text { leads to fast detection process for the } \\
\text { given mammogram. }\end{array}$ & $\begin{array}{l}\text { Dividing dataset into training and testing sets } \\
\text { after augmentation which may result in unfair } \\
\text { distribution for both sets. }\end{array}$ \\
\hline$[23]$ & $\begin{array}{l}\text { YOLO results in good detection } \\
\text { performance achieved in fast time. } \\
\text { The proposed approach can localize } \\
\text { masses that can exist in the dense parts in } \\
\text { pectoral muscles. }\end{array}$ & Small training and testing sets \\
\hline$[24]$ & $\begin{array}{l}\text { Using various classifiers to obtain the } \\
\text { best performance among them. } \\
\text { Fast detection and classification time in } \\
\text { less than } 0.025 \text { seconds. }\end{array}$ & $\begin{array}{l}\text { Limited sized training and the testing sets. } \\
\text { Not end to end system since the detection and } \\
\text { the classification are done separately which } \\
\text { needs the data to be prepared for each. }\end{array}$ \\
\hline$[25]$ & $\begin{array}{l}\text { Fast detection and classification in less } \\
\text { than } 9 \text { milliseconds due to utilizing } \\
\text { YOLO that looks at the whole image } \\
\text { once. } \\
\text { Using anchor boxes for training YOLO } \\
\text { generated from applying the k-means } \\
\text { clustering on the used dataset. }\end{array}$ & Small dataset. \\
\hline [26] & $\begin{array}{l}\text { Mammograms are pre-processed to } \\
\text { enhance their intensities which impact the } \\
\text { classification accuracy. } \\
\text { Features obtained from different layers of } \\
\text { VGG16 are concatenated to increase the } \\
\text { classification performance. }\end{array}$ & $\begin{array}{l}\text { No detection. } \\
\text { Low classification accuracy of } 76.0 \% \text {. }\end{array}$ \\
\hline
\end{tabular}

It is concluded from the comparative analysis that from the common advantages in proposing a CAD is developing an end-to-end system to be able to detect lesions in breast and classify its severity as well as done by [22]. Also, introducing a fast detection and classification process is one from the essential requirements nowadays to provide a real time detector results in fast decisions and diagnosis as achieved by [25]. From the disadvantages in the research of breast cancer detection field, is the low sized datasets and with accurate annotations at the same time. Recently, new datasets or updated ones have been published with accurate annotations and large number of samples. Training with large number of samples is very essential for good learning and fair results especially with the CNNs or the deep learning generally as for example in [20, 22]. 
As shown in Table 3 and Table 4, [19-21] used private datasets they obtained and with large number of samples. In [19], the mammograms are enhanced by using the median filters to remove noise results from the breast screening process. Then, the authors in [19] depend on segmenting the region of interest represent masses using some morphological operations to get $95.0 \%$. In [20], no pre-processing steps have been done except resizing mammograms to $224 * 224$ to be fit by the GMM which is used to allocate lesions and then segment them with accuracy of 96.0\%. While in [21], despite the existence of 8000 mammograms, the data is augmented by using different transformations as translating, rotation, flipping and scaling which one of the factors results in $97.52 \%$ which is better than $[19,20]$. Also, using CNNs in [21] is proved its ability to detect ROIs in more accurate manner than the traditional networks. Recently as shown starting from [22], the CNNs replaced the conventional networks due to its good performance and faster manner to learn. In [22], it is found that YOLO-V1 can be used as an end-to-end system to detect lesions in mammograms and then classify them results in detection accuracy of $99.7 \%$ and classification accuracy of $97.0 \%$. The data augmentation process from the conducted comparative analysis is proved its importance to be done for more effective training and more learned features that results in better results as implemented by the authors in [24-26].

Based on the compared recently proposed methods to detect lesions and classify them, it is conducted that the end-to-end models like YOLO and RetinaNet are the most suitable in case of performing both processes of the detection and classification. Since the end-to-end systems are faster in the applications required immediate results i.e., real time applications. As shown in Table 5, all are analysed to deduce the advantages and the disadvantages of each. As confirmed by Table 5 and the results in Table 3 and Table 4, it is proved that recently the CNNs are deployed to develop CAD systems since it obtains better results than the conventional mass detection models because they can extract features representing the lesions exist in mammograms without feature engineering [7, 8]. Based on the datasets comparison, we can conclude that the INbreast and the CBIS-DDSM are the most suitable datasets that can be used to localize lesions. Since the INbreast is the only existing FFDM and the CBIS-DDSM is the most accurate dataset that contains scanned film mammograms. Also, as shown in Table 3 and Table 4, it is very essential to pre-process mammograms to remove any extra artifacts to get better results.

\section{Conclusion and Future Work Directions}

During the previous years, the urgent need for developing CAD systems achieving high detection accuracy, have been increased due to the necessity of discovering the breast cancer early and the importance of the accurate diagnosis and treatment. Although the progress achieved by the recently proposed work discussed in this paper and others, there are still many challenges and directions remained to be resolved in the future research.

These challenges represented in detecting microcalcifications since most of the already existing methods care about revealing masses. Handling the high-resolution mammograms is considered a big challenge since most of the digital mammograms are of at least $2048 * 2560$. So, the models shall be updated to handle these high-resolution mammograms to see the fine details included in them. Most of the existing CAD works on X-rays mammograms, however there are other types of imaging modalities can be used like the MRI and ultrasound. Diagnosing the 3D mammograms instead of the 2D mammograms is also a challengeable point that required some investigation to utilize the $3 \mathrm{D}$ property to obtain higher detection and classification performance. 


\section{References}

1. M.A. Al-antari, M.A. Al-masni, S.U. Park, J.H. Park, M.K. Metwally, Y.M.Kadah, S.M. Han, T.-S. Kim, An automatic computer-aided diagnosis system for breast cancer in digital mammograms via deep belief network, J. Med. Biol. Eng. 38 (no. 3) (2017) 443-456.

2. Al-masni, Mohammed A., Mugahed A. Al-antari, J. M. Park, Geon Gi, Tae-Yeon Kim, Patricio Rivera, Edwin Valarezo, S-M. Han, and T-S. Kim. "Detection and classification of the breast abnormalities in digital mammograms via regional convolutional neural network." In 2017 39th Annual International Conference of the IEEE Engineering in Medicine and Biology Society (EMBC), pp. 1230-1233. IEEE, 2017.

3. Al-Masni, Mohammed A., Mugahed A. Al-Antari, Jeong-Min Park, Geon Gi, Tae-Yeon Kim, Patricio Rivera, Edwin Valarezo, Mun-Taek Choi, Seung-Moo Han, and Tae-Seong Kim. "Simultaneous detection and classification of breast masses in digital mammograms via a deep learning YOLO-based CAD system." Computer methods and programs in biomedicine 157 (2018): 85-94..

4. P. Boyle, B. Levin, et al., World cancer report 2008, IARC Press, International Agency for Research on Cancer, 2008.

5. Ukaoha, Kingsley Chiwuike, and Efosa Charles Igodan. "Architecture Optimization Model for the Deep Neural Network For Binary Classification Problems." International Journal of Intelligent Computing and Information Sciences (2020).

6. Ukaoha, Kingsley Chiwuike, and Efosa Charles Igodan. "Architecture Optimization Model for the Deep Neural Network." International Journal of Intelligent Computing and Information Sciences 19, no. 2 (2019): 1-16.

7. Al-furas, A., M. AL-dosuky, and Taher Hamza. "IMPROVING FEATURE MAPS IN EARLY LAYERS OF CONVOLUTIONAL NEURAL NETWORKS USING OTSU METHOD." International Journal of Intelligent Computing and Information Sciences 16, no. 2 (2016): 37-45.

8. Ali, H., M. Elmogy, E. ALdaidamony, and A. Atwan. "MRI BRAIN IMAGE SEGMENTATION BASED ON CASCADED FRACTIONAL-ORDER DARWINIAN PARTICLE SWARM OPTIMIZATION AND MEAN SHIFT." International Journal of Intelligent Computing and Information Sciences 15, no. 1 (2015): 71-83.

9. Wang, Y., Tao, D., Gao, X., Li, X. and Wang, B., 2011. Mammographic mass segmentation: Embedding multiple features in vector-valued level set in ambiguous regions. Pattern Recognition, 44(9), pp.1903-1915.

10. P. Rahmati, A. Adler, G. Hamarneh, Mammography segmentation with maximum likelihood active contours, Med. Image Anal. 16 (no. 9) (2012) 1167- 1186.

11. A.R. Domnguez, A. Nandi, Toward breast cancer diagnosis based on automated segmentation of masses in mammograms, Pattern Recognit. 42 (no. 6) (2009) 1138-1148.

12. Y. Qiu, S. Yan, R.R. Gundreddy, Y. Wang, S. Cheng, H. Liu, B. Zheng, A New approach to develop computer-aided diagnosis Scheme of breast mass classification using deep learning technology, J. X-Ray Sci. Technol. 25 (no. 5) (2017) 751-763.

13. Aouf, Mohamed. "Application of K-MTh Algorithm for Accurate Lunge Cancer Detection." International Journal of Intelligent Computing and Information Sciences 19, no. 2 (2019): 1-20.

14. Moreira, I. Amaral, I. Domingues, A. Cardoso, M. Cardoso, J. Cardoso, INbreast: toward a full-field digital mammographic database, Acad. Radiol. 19 (no. 2) (2012) 236-248.

15. Michael Heath, Kevin Bowyer, Daniel Kopans, Richard Moore and W. Philip Kegelmeyer, in Proceedings of the Fifth International Workshop on Digital Mammography, M.J. Yaffe, ed., 212218, Medical Physics Publishing, 2001. ISBN 1-930524-00-5. 
16. Rebecca Sawyer Lee, Francisco Gimenez, Assaf Hoogi , Daniel Rubin (2016). Curated Breast Imaging Subset of DDSM [Dataset]. The Cancer Imaging Archive.

17. Rebecca Sawyer Lee, Francisco Gimenez, Assaf Hoogi, Kanae Kawai Miyake, Mia Gorovoy \& Daniel L. Rubin. (2017) A curated mammography data set for use in computer-aided detection and diagnosis research. Scientific Data volume 4, Article number: 170177.

18. J Suckling et al (1994): The Mammographic Image Analysis Society Digital Mammogram Database Exerpta Medica. International Congress Series 1069 pp375-378.

19. Al-Masni, Mohammed A., Mugahed A. Al-Antari, Jeong-Min Park, Geon Gi, Tae-Yeon Kim, Patricio Rivera, Edwin Valarezo, Mun-Taek Choi, Seung-Moo Han, and Tae-Seong Kim. "Simultaneous detection and classification of breast masses in digital mammograms via a deep learning YOLO-based CAD system." Computer methods and programs in biomedicine 157 (2018): 85-94.

20. Kathale, Poonam, and Snehal Thorat. "Breast Cancer Detection and Classification." In 2020 International Conference on Emerging Trends in Information Technology and Engineering (icETITE), pp. 1-5. IEEE, 2020.

21. Al-Antari, Mugahed A., Seung-Moo Han, and Tae-Seong Kim. "Evaluation of deep learning detection and classification towards computer-aided diagnosis of breast lesions in digital X-ray mammograms." Computer methods and programs in biomedicine 196 (2020): 105584.

22. Mansour, Romany F. "A Robust Deep Neural Network Based Breast Cancer Detection and Classification." International Journal of Computational Intelligence and Applications 19, no. 01 (2020): 2050007.

23. Deb, Sagar Deep, Md Akhlaqur Rahman, and Rajib Kumar Jha. "Breast Cancer Detection and Classification using Global Pooling." In 2020 11th International Conference on Computing, Communication and Networking Technologies (ICCCNT), pp. 1-5. IEEE, 2020.

24. Al-masni, Mohammed A., Mugahed A. Al-antari, J. M. Park, Geon Gi, Tae-Yeon Kim, Patricio Rivera, Edwin Valarezo, S-M. Han, and T-S. Kim. "Detection and classification of the breast abnormalities in digital mammograms via regional convolutional neural network." In 2017 39th Annual International Conference of the IEEE Engineering in Medicine and Biology Society (EMBC), pp. 1230-1233. IEEE, 2017.

25. Khan, SanaUllah, Naveed Islam, Zahoor Jan, Ikram Ud Din, and Joel JP C. Rodrigues. "A novel deep learning based framework for the detection and classification of breast cancer using transfer learning." Pattern Recognition Letters 125 (2019): 1-6.

26. Aly, Ghada Hamed, Mohammed Marey, Safaa Amin El-Sayed, and Mohamed Fahmy Tolba. "YOLO Based Breast Masses Detection and Classification in Full-Field Digital Mammograms." Computer Methods and Programs in Biomedicine (2020): 105823. 